\title{
Editorial: Integrated Quantum Photonics
}

\author{
Shinichi Saito* \\ Center for Exploratory Research Laboratory, Research and Development Group, Hitachi, Ltd., Tokyo, Japan
}

Keywords: quantum random number generators, Si photonics integrated circuits, optical vortex, photonic crystal, poincare sphere, quantum photonic circuits

Editorial on the Research Topic

Integrated Quantum Photonics

Integration of electronic transistors in a silicon ( $\mathrm{Si}$ ) chip has now exceeded more than $10 \mathrm{~B}$ in microprocessing units (MPUs) and graphics processing units (GPUs), which is even larger than the number of neurons in a human brain. The extensive usages of these processing units for various machine-learning and artificial intelligence (AI) applications also demand integration of photonic devices for Internet-Of-Things (IOT) networks and big data analysis. Si photonics will be a key technology for photonics integration, and for the future, photonic-based quantum technologies will be introduced. One of the obvious advantages to use photons instead of electrons for quantum technologies is the potential to introduced these devices at room temperatures without complicated expensive cryogenic systems. We thought it is a good time to call for papers in integrated quantum photonics in Frontiers. In this research topic, five papers were submitted, one paper was rejected, four papers were accepted, but one paper was retracted by the authors. The number of papers which we have attracted was smaller than we expected, but we think the research area will grow over the years.

In the paper of Bisadi et al. a compact quantum random number generator was

OPEN ACCESS

Edited and reviewed by:

Lorenzo Pavesi,

University of Trento, Italy

*Correspondence: Shinichi Saito shinichi.saito.qt@hitachi.com

Specialty section:

This article was submitted to Optics and Photonics,

a section of the journal Frontiers in Physics

Received: 23 October 2021 Accepted: 28 October 2021 Published: 26 November 2021

Citation:

Saito S (2021) Editorial: Integrated Quantum Photonics.

Front. Phys. 9:800648.

doi: 10.3389/fphy.2021.800648 demonstrated, using a novel light-emitting diode using Si nanocrystals. The group, led by Pavesi, is a leading group for light emissions from Si nanocrystals, and a random number generator will be a suitable application, relying on quantum mechanical probabilistic characteristics of spontaneous emissions. They have developed an integrated system together with an $\mathrm{Si}$ photomultiplier and a field-programmable gate array (FPGA) processing unit and showed that their data pass all statistical standards as random numbers. The recorded bit rate of $0.5 \mathrm{Mbps}$ is already comparable to commercial products without using Si nanocrystals. The technology with Si nanocrystals will be used for applications which require even more severe security controls, such as secure communications, among governments, mobile banking, and statistical research.

For various quantum applications, we need a two-level system for describing a quantum bit (qubit). In the paper of Sotto et al. they found a new way to control a polarization state as a two-level system by introducing a phase mismatch between adjacent photonic crystals. In a standard design of a waveguide in a photonic crystal, a line defect is introduced simply by removing holes for a waveguide, and the parity symmetry against mirror reflection is maintained. They intentionally introduced a mismatch by introducing a phase shift among adjacent photonic crystals across the waveguide, which allowed coupling of two propagation modes. By applying a simple model for a twolevel system, they found the energy gap is opening up by controlling the amount of the phase shift.

In the last paper, another two-level system is proposed by using photons with left and right vortices. Photons with vortices have optical orbital angular momentum (OAM), and we expect a quantum mechanical superposition state among left and right circulations in a hyper- 
Poincaré sphere. By using a simple Si photonic two-bus waveguide together with a complex micro-gear, the amplitudes and phases are controlled for the left and right vortices. The device was named a Poincaré rotator, since it allows to rotate the vectorial OAM state in a hyper-Poincaré sphere. The conservation law of spin and OAM was discussed.

We believe the research area of integrated quantum photonics will be even more important for the future, and proposed devices will be developed beyond original foreseeable applications.

\section{AUTHOR CONTRIBUTIONS}

The author confirms being the sole contributor of this work and has approved it for publication.

\section{FUNDING}

The author SS is supported by JSPS KAKENHI Grant Number JP $18 \mathrm{~K} 19958$.

Conflict of Interest: SS is employed by Hitachi.

Publisher's Note: All claims expressed in this article are solely those of the authors and do not necessarily represent those of their affiliated organizations, or those of the publisher, the editors and the reviewers. Any product that may be evaluated in this article, or claim that may be made by its manufacturer, is not guaranteed or endorsed by the publisher.

Copyright (c) 2021 Saito. This is an open-access article distributed under the terms of the Creative Commons Attribution License (CC BY). The use, distribution or reproduction in other forums is permitted, provided the original author(s) and the copyright owner(s) are credited and that the original publication in this journal is cited, in accordance with accepted academic practice. No use, distribution or reproduction is permitted which does not comply with these terms. 